\title{
Author Correction: Sphingosine 1-phosphate modulation and immune cell trafficking in inflammatory bowel
}

Bram Verstockt (D), Stefania Vetrano, Azucena Salas (D), Shadi Nayeri, Marjolijn Duijvestein (D), Niels Vande Casteele (i) and Alimentiv Translational Research Consortium (ATRC)

Correction to: Nature Reviews Gastroenterology \& Hepatology https://doi.org/10.1038/s41575-021-00574-7, published online $14^{\text {th }}$ February 2022.

In the original version of this article, the affiliation details for Silvio Danese were incorrect. They have now been corrected to 'Gastroenterology and Endoscopy, IRCCS Ospedale San Raffaele, Milan, Italy' and 'University Vita-Salute San Raffaele, Milan, Italy'. The numbering of the affiliations for the Alimentiv Translational Research Consortium (ATRC) was updated as a result of these changes. These errors have now been corrected in the HTML and PDF versions of the article.

https://doi.org/10.1038/s41575-022-00597-8 I Published online 7 March 2022

๑) Springer Nature Limited 2022 